\title{
Stearoyl-CoA desaturase 1 is a novel molecular therapeutic target for clear cell renal cell carcinoma
}

\author{
Christina A. von Roemeling ${ }^{1}$, Laura A. Marlow ${ }^{1}$, Johnny J. Wei ${ }^{1}$, Simon J. Cooper ${ }^{1}$, \\ Thomas R. Caulfield ${ }^{2}$, Kevin $\mathbf{W u}^{3}$, Winston W. Tan ${ }^{4}$, Han W. Tun ${ }^{4}$, and John A. Copland ${ }^{1,{ }^{*}}$ \\ ${ }^{1}$ Department of Cancer Biology, Mayo Clinic Comprehensive Cancer Center \\ ${ }^{2}$ Dept. of Neuroscience \\ ${ }^{3}$ Department of Laboratory Medicine and Pathology \\ ${ }^{4}$ Division of Hematology \& Oncology
}

\begin{abstract}
Purpose-We set out to identify SCD1 as a novel molecular target in clear cell renal cell carcinoma (ccRCC) and examine its role in tumor cell growth and viability in vitro and in vivo independently as well as in combination with current FDA approved regimens.

Experimental Design-Patient normal and ccRCC tissue samples and cell lines were examined for SCD1 expression. Genetic knockdown models and targeted inhibition of SCD1 through use of a small molecule inhibitor, A939572, were analyzed for growth, apoptosis, and alterations in gene expression using gene array analysis. Therapeutic models of synergy were evaluated utilizing pharmacologic inhibition of SCD1 with the tyrosine kinase inhibitors (TKI) sunitinib and pazopanib, and the mTOR inhibitor temsirolimus.
\end{abstract}

Results-Our studies identify increased SCD1 expression in all stages of ccRCC. Both genetic knockdown and pharmacologic inhibition of SCD1 decreased tumor cell proliferation and induced apoptosis in vitro and in vivo. Upon gene array, quantitative real-time PCR, and protein analysis of A939572 treated or SCD1 lentiviral knockdown samples, induction of endoplasmic reticulum (ER) stress response signaling was observed, providing mechanistic insight for SCD1 activity in ccRCC. Furthermore, combinatorial application of A939572 with temsirolimus synergistically inhibited tumor growth in vitro and in vivo.

Conclusions-Increased SCD1 expression supports ccRCC viability and therefore we propose it as a novel molecular target for therapy either independently or in combination with an mTOR inhibitor for patients whose disease cannot be remedied with surgical intervention, such as in cases of advanced or metastatic disease.

\section{Keywords}

Stearoyl-CoA desaturase 1; clear cell renal cell carcinoma; metabolism; mTOR; antitumor synergy

\section{Introduction}

Currently ccRCC is the most common subtype of RCC, which is the most prevalent kidney cancer among adults, accounting for approximately $89 \%$ of all diagnosed cases. Incidence of

Correspondence: John A. Copland, Ph.D. Dept. of Cancer Biology, Mayo Clinic Comprehensive Cancer Center, 4500 San Pablo Road, Jacksonville, Florida, 32224. Phone: 904-953-6120; Fax: 904-953-0277; copland.john@ mayo.edu.

Conflicts of interest: The authors of this manuscript have no conflicts of interest to disclose. 
RCC has been increasing steadily in the United States although mortality rates appear to have stabilized, likely due to early diagnosis among patients (1). Average age of diagnosis occurs typically between 60 and 64 years of age, though cases have been reported in younger individuals. Localized disease yields better patient outcomes as these cases can be addressed by partial or whole nephrectomy resulting in extended disease-free survival with $90 \%$ of stage I and $51 \%$ of stage II patients having an estimated five year or greater survival rate (2). For those patients diagnosed with advanced or metastatic disease the prognosis is markedly worse, with median survival periods dropping to approximately 3.1 years and 1.1 years for stage III and stage IV patients respectively (2). For individuals presenting with advanced disease, treatment options are limited with no current drug therapy leading to long term survival (3) with the exception of 6-7\% of patients who respond to interleukin-2 (4).

FDA approved options for treatment of advanced ccRCC includes cytokine immunotherapy (interleukin-2, interferon) $(4,5)$ as well as treatments involving agents that target pathways believed to facilitate ccRCC progression such as angiogenesis and mTOR signaling (6). TKI's such as sunitinib (Sutent $\left.{ }^{\circledR}\right)(7)$, pazopanib (Votrient $\left.{ }^{\circledR}\right)(8)$, sorafenib (Nexavar $($ ) $)(9)$, and axitinib (Inlyta $\left.{ }^{\circledR}\right)(10)$ block angiogenesis though inhibition of VEGFR and PDGFR. While there are treatments available for advanced ccRCC, none are curative and drug resistance occurs in all cases, often manifesting in more aggressive disease. This presents the need for application of multi-targeted therapy; however current options may increase sideeffects due to drug toxicity which prohibit their use in combinatorial therapy $(3,5)$. For example, a phase I clinical trial investigating the combination of sunitinib with temsirolimus produced significant toxicity even at low doses, and further investigation was terminated (11). In a phase II randomized study combining temsirolimus with bevacizumab, this treatment not only generated an increase in toxicity which limited treatment continuation, but also did not demonstrate improvement of progression free survival (12). There is a clear need for the identification of alternative treatments, with an emphasis on tumor specific targeting in order to minimize side effect complications.

At present, there is a deficiency in the elucidation of the hallmark genetic features which contribute to ccRCC. Loss of VHL and resulting stabilization of hypoxia-inducible factors (HIF-1 $a$ and HIF-2 $a$ ) is one of the few accepted signatures correlated to ccRCC (5). Causative somatic mutations of other well studied oncogenic and tumor suppressive factors including KRAS, HRAS, NRAS, PI3K, EGFR, BRAF, MYC, TP53 and PTEN comprise less than $1 \%$ of all ccRCC cases (13). Despite these shortcomings, our previous studies examining the genetic signature of ccRCC have identified aberrant metabolic regulation as contributory to tumor growth and survival, including errant expression of nutrient sensing and processing machinery (14), and this may provide an avenue for development of therapeutic intervention.

SCD1, a constituent of lipid metabolism, was observed by us to be abnormally expressed in ccRCC at high levels when compared to patient matched normal tissue both at the transcriptional and protein level. This occurs as an early event seen in stage I patient samples, and expression remains elevated throughout the course of disease progression as it is observed in all other stages of ccRCC in nearly all patient tumor samples while normal tissue expression remained nominal if at all present. SCD1 is an iron-containing enzyme belonging to a family of fatty acyl desaturases, whose role is to catalyze the biosynthesis of $\Delta 9$ monounsaturated fatty acids (MUFA), oleic and palmitoleic acid, from the saturated fatty acids (SFA) stearic and palmitic acid. It is a critical enzyme in the fatty acid metabolism pathway and is a rate limiting step in MUFA synthesis $(15,16)$. MUFAs are involved in many biological processes and are a major constituent of biological structures such as membranes, and can also function as or modify signaling molecules (15-17). This 
suggests a potential higher need for them in dynamic or rapidly dividing cells such as cancer cells.

In this report, we demonstrate overexpression of SCD1 in patient ccRCC tissue, and investigate its role in tumor viability through manipulation of genetic expression and by pharmacologic inhibition. Resulting analysis of tumor cell proliferation, viability, and gene signaling demonstrated that loss of SCD1 activity blocked cell growth, induced apoptosis, and promoted ER stress. In an effort to develop a multi-targeted approach to compromise ccRCC perpetuation, we investigated the effects of using A939572 in combination with current FDA approved regimens. Herein, we demonstrate antitumor synergy in vitro and in vivo combining A939572 with an mTOR inhibitor.

\section{Materials and Methods}

\section{Chemicals}

Temsirolimus, pazopanib and sunitinib were purchased from Selleck Chemicals Co. Ltd. A939572 was purchased from BioFine International.

\section{Cell Culture}

ccRCC cell lines: RWV366T and KIJ265T (18) (both stage IV ccRCC patient tissue derived, Copland laboratory, Mayo Clinic, Florida), A498, Caki1, Caki2, and ACHN (ATCC) and K347N, K355N, K359N, K360N, K365N, and K366N normal renal tissue derived mortal cells (NRE) were cultured in DMEM medium (Cellgro) containing 5\%FBS (Hyclone) and $1 \times$ penicillin-streptomycin (Invitrogen) at $37^{\circ} \mathrm{C}$ in humidified conditions with $5 \% \mathrm{CO}_{2}$.

\section{Growth Assays}

Cells were plated $\left(0.5\right.$ or $1 \times 10^{5} /$ well $)$ in 24 -well plates (Midwest Scientific) in triplicate. Cells were counted using a Coulter Particle Counter (Beckman). Oleic acid-albumin (Sigma Aldrich) was added to media at $5 \mu \mathrm{Mol}$. Drug stocks were prepared in DMSO (Sigma). Temsirolimus dosing was performed as described in the text. Soft agar cultures were prepared by diluting $2 \times$ growth medium $1: 1$ in $1.5 \%$ Seaplaque ${ }^{\circledR}$ GTG ${ }^{\circledR}$ agarose (Lonza), with 500 cells/plate in $60 \mathrm{~mm}$ culture dishes (Genesee Scientific). Colonies were stained with Giemsa (LabChem Inc.) and counted after 3 wks.

\section{Lentivirus}

MISSION shRNA pLKO.1 constructs (Sigma-Aldrich) were used to make self-inactivating shRNA lentiviruses for human SCD1 (clones: NM_005063.3-1200s1c1 [shSCD1200], NM_005063.3-780s1c1[shSCD780]), human ATF6(NM_007348.1-332s1c1[shATF6-332]), and a non-target (NT) random scrambled sequence control (SHC002). Transfection reagents Lipofectamine 2000 (Invitrogen) and ViraPower (Invitrogen) were used to generate lentiviruses using HEK293FT viral progenitor cells (Invitrogen). ccRCC and NRE cells were incubated with lentivirus plus $5 \mu \mathrm{g} / \mathrm{mL}$ polybrene (American Bioanalytical) for $24 \mathrm{hrs}$ prior to clonal selection with Puromycin (Fischer).

\section{Transfections and Luciferase Assays}

Caki1 and A498 cells were transiently transfected with p5xATF6-GL3 UPR luciferase reporter [Addgene (plasmid\#11976)] and pRL-CMV-renilla luciferase plasmid (Promega) using Lipofectamine2000 (Invitrogen). Cells (DMSO vs. A939572, NT vs. shSCD780) were harvested after 48hrs using Promega's Dual Luciferase assay kit per the manufacturer's 
protocol and luciferase activity was measured using a Veritas Luminometer (Promega); reported as relative luminescence.

\section{RNA Isolation and Quantitative PCR}

RNA isolation, preparation of cDNA, and QPCR was performed as previously described (14). TaqMan®FAM ${ }^{\mathrm{TM}}$ dye-labeled probes including POLR2A (Hs00172187_m1normalization control), SCD1(Hs01682761_m1), HSPA5(Hs99999174_m1), CEBP $\beta($ CEBPB Hs00270923_s1), GADD45A(Hs00169255_m1), DDIT3(Hs01090850_m1), HERPUD1(Hs01124269_m1), and ATF6(Hs00232586_m1). Fold change value comparisons: normal vs. tumor, NT vs. target lentivirus, and DMSO vs. A939572 treated samples using the $\Delta \Delta \mathrm{Ct}$ method (19).

\section{Gene Array Expression Analysis}

Mayo Clinic Advanced Genomic Technology Center Gene Expression Core performed gene array using Affymetrix Human Genome U133 Plus 2.0 Array chip. Data processing and methodology are as previously described (14). Gene expression data is deposited at Gene Expression Omnibus (Accession\#GSE41485). Pathway analysis was performed using IPA (Ingenuity® Systems).

\section{Western Blot Analysis}

Protein extraction and western blot analysis was performed as previously described (20). Primary antibodies included SCD1 (Sigma-Aldrich), PARP (Cell Signaling), DDIT3 (Cell Signaling), BiP (Cell Signaling), sXBP1 (Santa Cruz Biotechnology), and $\beta$-actin (SigmaAldrich).

\section{IHC and ICC Analysis}

Samples include formalin-fixed, paraffin-embedded tissue microarray (TMA) of patient ccRCC tumor plus matched normal tissues and combinatorial in vivo mouse tumor tissue. Samples were mounted on slides, blocked with Diluent (Dakocytomation) for 30min, and then probed as specified in text for SCD1, Ki67 (Invitrogen), Caspase-3 (Cell Signaling), CD31 (Santa Cruz Biotechnology), phospho-mTOR (Cell signaling), DDIT3, and XBP1. ICC preparation and staining was performed as previously described (18). Stain scoring was done using algorithms generated with Imagescope software (Aperio) created by a histologist. H-scores were calculated based upon signal intensity (0-3+) using the formula: $\left[\left(1^{+} \% \times 1\right)+\left(2^{+} \% \times 2\right)+\left(3^{+} \% \times 3\right)\right]$, intensity (I)-scores were calculated by dividing signal intensity by area, and nuclear $(\mathrm{N})$-scores were calculated by dividing $\%$ positive nuclei by total nuclei examined per area. Cases where insufficient tumor tissue presented were excluded. 20x images were obtained using Scanscope XT and Imagescope software. This study was approved by the Mayo Institutional Review Board. RWV366T cell line validation was carried out as previously described (18).

\section{In Vivo Analysis}

A498 cells were subcutaneously implanted in athymic nu/nu mice (Harlan Laboratories) at $1 \times 10^{6}$ cells/mouse in 50\%Matrigel (BD Biosciences). Tumors reached $\sim 50 \mathrm{~mm}^{3}$ prior to 4 wk treatment. A939572 was re-suspended in strawberry flavored Kool-Aid® in sterilized $\mathrm{H}_{2} \mathrm{O}(0.2 \mathrm{~g} / \mathrm{mL})$ vehicle at $30 \mathrm{mg} / \mathrm{kg}$ in a $50 \mu \mathrm{l}$ dose. Mice were orally fed by using a syringe to administer the $50 \mu 1$ dose twice daily/mouse. This modified method was found to be effective and less stressful on the mice. Temsirolimus was solubilized in $30 \%$ ethanol/saline and administered via intraperitoneal injection at $10 \mathrm{mg} / \mathrm{kg}$ in a $50 \mu \mathrm{l}$ dose once every $72 \mathrm{hrs} /$ mouse. Tumor volumes were calculated using the formula $0.5236\left(\mathrm{~L}^{*} \mathrm{~W} * \mathrm{H}\right)$ and body weight were measured every 3 days. 


\section{DNA isolation and STR Analysis}

Genomic DNA was extracted from both RWV366T patient primary tissue and matching cell line using Purelink ${ }^{\mathrm{TM}}$ Genomic DNA mini kit (Invitrogen). Sixteen STR markers were PCR amplified using fluorescently labeled primers from ABI (Applied Biosystems), and were analyzed using ABI 3130 (Applied Biosystems). Peak sizes were calculated versus a coinjected size standard using Gene Marker (Soft Genetics, State College, PA).

\section{Statistical Analysis}

Data values are presented as either percentage or fold change \pm s.d. unless otherwise specified. Fold change values $1.5<$ are considered statistically significant. Treatment group comparisons were analyzed using two-tailed paired Student's $t$-test with $\mathrm{p}<0.05$ being considered statistically significant. Statistically significant results are indicated by asterisk (*). Drug synergy statistics are indicated via combination index (CI) determined using CalcuSyn ${ }^{\circledR}(21)$ as described in the text.

\section{Results}

\section{SCD1 is upregulated in clear cell renal cell carcinoma}

Quantitative real time PCR (QPCR) of total mRNA extracted from patient stage I through IV as well as metastatic ccRCC tumor tissues and matched distant site normal tissue was analyzed for gene expression of $S C D 1$, and was found to be consistently upregulated in all tumor samples examined when compared to matched normal samples (Figure 1A). Similarly, immunohistochemical (IHC) staining for SCD1 protein expression in patient matched tumor and normal tissue confirmed elevated expression in tumor samples across all stages (Figure 1B). Increased SCD1 expression in metastatic samples is confirmed by western blot (Figure 1B).

For purposes of identifying relevant cell line models to utilize in further experimentation, QPCR and western blot analysis of six primary NRE and six ccRCC cell lines for SCD1 expression (Figures 1C,D) was performed and yielded expression patterns analogous to those observed in tissue. NRE samples did express some variability when compared to the lowest mRNA value exhibited by $366 \mathrm{~N}$, likely reflective of normal biological fluctuations; however they remained significantly lower than tumor values. RWV366T is a newly established patient derived ccRCC cell line, whose patient and renal origins are validated by STR analysis and IHC for renal markers in Sup. Figure 1A,B.

\section{SCD1 is required for CCRCC tumor cell growth and survival}

In order to identify the role of increased SCD1 in regards to ccRCC viability as well as validate specificity in tumor compared to normal samples, two lentiviral constructs designed to target SCD1 (shSCD780 and shSCD1200) were used to knock down SCD1 expression in two NRE (K359N, K360N) and two ccRCC cell lines (Caki1, A498). K360N was included as a NRE control as it demonstrated protein expression of SCD1. QPCR analysis for SCD1 expression confirmed over $80 \%$ mRNA silencing in all samples relative to NT controls for each cell line (Figure 2A). Specificity of shSCD780 and shSCD1200 for SCD1 was confirmed by western blot (Figure 2D). Subsequent growth analysis resulted in over an $80 \%$ decrease in proliferation among tumor samples at day 5 post-infection (Figure 2B) but not in NRE samples (Figure 2C). Because such a considerable proliferative decrease in tumor cells was observed, western blot analysis for poly-ADP-ribose polymerase (PARP) cleavage, a marker for apoptosis, was performed. Results confirmed PARP cleavage in both Cakil and A498 cells infected with each shSCD lentiviral construct compared to NT controls (Figure 2D), and indicates that loss of proliferation is due in part by induction of programmed cell death. 


\section{Oleic Acid reverses effects of decreased SCD1 expression in tumor cells}

As oleic acid (OA) is the principle product of SCD1 mediated SFA dehydrogenation (15), a cell culture stable form of OA conjugated to albumin from bovine serum (OA-BSA) was utilized to perform rescue experimentation in order to confirm that decreased tumor cell growth and induction of cell death was due to lentiviral mediated suppression of SCD1.

Media alone and BSA served as control groups. Proliferation assay of NT control versus shSCD780 infected Caki1 and A498 cells supplemented with or without OA-BSA were counted after five days. Both Caki1 and A498 shSCD780 cells exhibited significant decreases in growth when compared to controls; however the addition of OA-BSA rescued the proliferative capacity of these cells to near control rates (Figure 3A). Notably, addition of OA-BSA to Caki1 NT cells marginally enhanced proliferation (Figure 3A). SCD1 knockdown by lentiviral infection was confirmed at the protein level (Figure 3B). In addition to growth rescue, supplementation with OA-BSA also decreased shSCD780 induced apoptosis as demonstrated by reduction in PARP cleavage shown by western blot (Figure 3B). Representative phase contrast images of ccRCC cells for each group are shown in Figure 3C.

\section{Small molecule inhibition of SCD1 induces ccRCC cell death}

A939572 is a small molecule that specifically inhibits SCD1 enzymatic activity (Sup. Figure 2A) (22). A939572 demonstrated a significant dose-dependent decrease in proliferation in Caki1, A498, Caki2, and ACHN at day 5 (IC50s of 65nM, 50nM, 65nM, and 6nM respectively) (Figure 4A). Molecular target specificity was confirmed by addition of OABSA to the growth inhibitory assay, with IC50 doses applied to all four cell lines versus DMSO+BSA control. Addition of OA-BSA prevented A939572 mediated growth inhibition, comparable to control groups in all four cell lines (Figure 4B). In congruity with previous experimentation examining SCD1 lentiviral knockdown models, A939572 induced apoptosis confirmed by PARP cleavage via western blot analysis in all four cell lines (Figure 4C). Addition of OA-BSA blocked apoptosis noted by lack of PARP cleavage (Figure 4C). Representative phase contrast cell images (Figure 4D) demonstrate marked reduction in confluence of $\mathrm{A} 939572$ treated ccRCC cells, which reflects decreased proliferation and induction of cell death as a result of treatment, where OA-BSA supplemented cells display no visible alterations in phenotype. Thus, we have identified a specific small molecule SCD1 inhibitor that induces apoptotic cell death that can be rescued by oleic acid.

\section{Treatment of ccRCC cells with A939572 induces Endoplasmic Reticulum Stress}

In order to determine the mechanism of decreased proliferation and induction of cell death associated with loss of SCD1 activity in ccRCC cells, gene array analysis was performed with Caki1, A498, Caki2, and ACHN ccRCC cells treated for 24 hours with a 75nM dose of A939572 compared to DMSO control. Gene expression data was analyzed using the Ingenuity ${ }^{\circledR}$ Systems (IPA) program and revealed increased expression of ER stress response genes associated with UPR (23). A summary of ER stress genes induced by A939572 treatment is listed along with median fold change induction in Sup. Figure 2B.

Western blot of Caki1 and A498 cells for protein expression of ER stress markers including $\mathrm{BiP}$ (heat shock 70kDa protein, GRP78), CHOP (DNA damage inducible transcript 3, DDIT3), and spliced-XBP1 (x-box binding protein 1, s-XBP1) revealed amplified expression in both drug treated $(75 \mathrm{nM})$ and shSCD780 lentiviral knockdown cells after 48 hours (Figure 5A), confirming induction of ER stress upon loss of SCD1 activity or expression as implicated by the gene array analysis. 
In order to validate the specificity of ER stress induction mediated by both A939572 and shSCD780, rescue assays were performed using OA-BSA in Caki1 and A498 cells. QPCR analysis of five ER stress genes identified in the gene array including BiP, CHOP, HERPUD1 (homocysteine-inducible, ER-stress inducible, ubiquitin-like-1), GADD45a (DNA damage inducible transcript 1, DDIT1), and CEBP $\beta$ (CCAAT/enhancer binding protein beta) were examined. In A939572 (SCDi) treated Caki1 and A498 cells, all five ER stress related genes were expressed at significantly increased levels compared to DMSO +BSA control, and this elevated expression could be blocked with the addition of OA-BSA (Figure 5B). In shSCD780 lentiviral infected Caki1 and A498 cells, all of the ER stress genes were significantly induced in the Caki1 shSCD780 sample and 4 of the 5 were significantly induced in the A498 shSCD780 sample. Similar to the drug treated cells, OABSA successfully blocked shSCD780 induced expression of the ER stress genes (Figure $5 B)$.

ER stress induction due to genetic and pharmacologic inhibition of SCD1 was assessed in NRE cells. Since A939572 did not yield a dose response in NRE cells (Sup. Figure 2C), a dose of 100nM was applied for 48 hours. No significant ER stress induction, measured by QPCR for ER stress markers CHOP, HERPUD1, GADD45a, and BiP, was observed in K359N utilizing either lentiviral(shSCD780) or A939572 SCD1 inhibition (Sup. Figure 2D). Interestingly, lentiviral inhibition of SCD1 in K360N cells, which do demonstrate SCD1 protein expression (Figure 1D), yielded no induction of ER stress, however A939572 treatment led to a slight induction of ER stress as two of the four ER stress markers evaluated, HERPUD1 and BiP, were upregulated $\sim 2$ fold (Sup. Figure 2D). Nevertheless, the ER stress induction observed in A939572 treated $\mathrm{K} 360 \mathrm{~N}$ is milder in comparison to QPCR values of ER stress induction observed in A498 and Cakil ccRCC cells (Figure 5B), and neither A939572 or lentiviral inhibition of SCD1 in K360N led to attenuated cell proliferation in K360N (Figure 2C, Sup. Figure 2C). Thus, ER stress is strongly induced in SCD1 expressing ccRCC cell lines.

Activating transcription factor 6 (ATF6) is a key bZIP transcription factor that mediates part of the UPR stress response $(24,25)$. Upon stress induction, ATF6 incites activation of many downstream mediators in the ER stress response pathway including XBP1, BiP, HSP9OB1 (heat shock protein 90kDa beta), GADD45a, HERPUD1 and $C H O P(25,26)$. Cakil and A498 cells transfected with an ATF6 luciferase reporter (p5xATF6-GL3) (24) were treated with A939572 or were infected with shSCD780. Inhibition of SCD1 resulted in significant enhancement of luciferase activity as compared to DMSO and NT controls, where the addition of OA-BSA significantly reduced reporter activation (Sup. Figure 3). This confirms that ATF6 is activated by inhibition of SCD1 activity in ccRCC cells.

In order to deduce whether ER stress directly mediates the effects on proliferation and cell death observed exclusively in ccRCC cell lines due to SCD1 inhibition (Figures 2-4), ATF6 was silenced using a lentiviral construct (shATF6-332) in Caki1 and A498 ccRCC cells. Effects on ER stress induction and proliferation were evaluated in the presence of A939572. QPCR for ATF6 expression exhibited attenuation of over 85\% in both Caki1 and A498 shATF6-332 cells both in DMSO and A939572 treated groups when compared to NT DMSO controls (Figure 5C). QPCR analysis for ER stress markers regulated by activation of ATF6 (CHOP, HERPUD1, and GADD45a) was performed. Transcript expression levels displayed that knockdown of ATF6 blocked A939572 mediated induction of CHOP, HERPUD1, and GADD45a in both cell lines, and in fact decreased levels of HERPUD1 in Cakil cells, and CHOP in both cell lines (Figure 5C). This suggests that ATF6 knockdown successfully compromised the induction of the ER stress response in ccRCC. Proliferation assay of NT and shATF6-332 in DMSO vs. A939572 demonstrated that ATF6 suppression rescued decreased proliferation observed in NT samples due to treatment with A939572 in 
both Caki1 and A498 cells (Figure 5D). This data supports that loss of ccRCC viability via inhibition of SCD1 is conducted through the ER stress response.

\section{Combination of A939572 with Temsirolimus synergistically enhances tumor cell death}

In order to target ccRCC using a multifaceted approach, synergy was examined through application of combinatorial treatment utilizing A939572 in congruence with a current FDA approved regimen for ccRCC treatment. These included the TKIs pazopanib and sunitinib, as well as the mTOR inhibitor temsirolimus.

After identifying appropriate cell proliferative dose responses for pazopanib and sunitinib in four ccRCC cell lines including A498, Caki1, Caki2, and ACHN (Sup. Figure 4A,C), both TKIs were dosed in combination with A939572 up to approximately the IC50 dose for each drug in the Caki1 and the A498 cell lines. No synergy was noted in either Caki1 or A498 cells with combinatorial treatment (Sup. Figure 4B,D). No dose response could be determined for Temsirolimus(Tem) (Sup. Figure 5). Combinatorial treatments were therefore done using a fixed dose of Tem $(0.1 \mathrm{nM}, 1 \mathrm{nM}$, and $10 \mathrm{nM})$ combined with a dose range of A939572 up to the IC50 in Caki1, A498, Caki2 and ACHN cells. Both drugs in combination yielded very strong synergy in all four cell lines (Sup. Figure 6A-D) as indicated by the combination index (CI) determined using CalcuSyn ${ }^{\circledR}(21)$ based on the Chou-Talalay Method where CI values $>1$ represent an antagonistic effect and values $<1$ represent synergy, with lower values signifying enhanced synergy (27). Colony formation assay of A498 cells grown in soft agar treated with mono and combination doses of $5 \mathrm{nM}$ A939572 and 5nM Tem (Sup. Figure 7) reflected synergistic effects observed in combination proliferation assays, and provided the rationale for in vivo analysis of combinatorial therapy.

Athymic nude (nu/nu) mice bearing A498 ccRCC xenografts were treated with A939572 and Tem individually or in combination over the course of four weeks, and tumor volume $\left(\mathrm{mm}^{3}\right.$ ) was recorded (Figure 6A). A939572 and Tem monotherapy generated similar growth responses with approximately 20-30\% reductions in tumor volume (vs. placebo control) being observed upon study completion, with values reaching statistical significance only within the last week of treatment. The combination group yielded over a $60 \%$ decrease in tumor volume (vs. placebo control) by study completion with significant reductions recorded after approximately 1 week of treatment. All of the animals maintained a healthy weight throughout the course of the treatment (Figure 6A), however those in both the A939572 and the Combo group exhibited increased blinking, and slight mucosal discharge from the eyes after the first week of treatment.

IHC analysis of tumors resected from each treatment group was analyzed for proliferation, angiogenesis, and cell death (Figure 6B). All treatment groups (A939572, Tem, and Combo) when compared to the placebo control exhibited decreased proliferation as marked by reduction in percent positivity of nuclear Ki67 staining, with the combinatorial group demonstrating the most significant decline. Angiogenesis as examined by intensity of microvessel density demonstrated a slight decrease in both the Tem and the Combo groups; however the cumulative scores were not considered significant. Cell death as examined by cleaved caspase-3 (CC3) demonstrated significant increases in the Combo group when compared to all groups. A moderate increase in cell death was also seen in the A939572 and Tem groups compared to the placebo. Phosphorylated mTOR was inspected as a marker for temsirolimus activity, and decreased expression was confirmed in both the Tem and the Combo groups as compared to the Placebo group. ER stress was examined via western blot of total protein extractions prepared from randomly selected tumor tissue samples representing each treatment group, and resulting quantitative expression was normalized to respective $\beta$-actin controls. Increased expression of $\mathrm{CHOP}$ was confirmed in all samples 
treated with A939572 (A939572 and Combo) (Figure 6C) confirming that inhibition of SCD1 in ccRCC contributes to ER stress in vivo. Interestingly, samples in the Tem group also exhibited induction of CHOP, although to a lesser extent when compared to A939572 and Combo groups. Temsirolimus has been previously reported to decrease SCD1 expression in breast cancer cells (28). Inhibition of mTOR in ccRCC could indirectly mediate ER stress through decrease of SCD1, thereby explaining our observations. Little to no CHOP expression was seen in any placebo samples. In summary of our cumulative findings, we propose that attenuation of SCD1 activity by either genetic knockdown or small molecule inhibitors promotes ER stress through accumulation of SFA species as depicted in Figure 6D via a mechanism that is conversed in detail in the following discussion.

\section{Discussion}

Despite current advances in cancer research which have improved our understanding of cancer cell biology, there are still limitations in current therapeutic options for patients with advanced ccRCC $(3,6)$. Two primary explanations for this include the lack of molecular targets in ccRCC which manifest consistently in a significant proportion of patient tumor samples. Also, the adaptive nature of tumor cells typically results in acquired drug resistance with selective pressure sometimes yielding outgrowth of more malignant subpopulations (29-31). It is clear that development of more effective therapeutic strategies must encompass identification of molecular targets as well as employing multi-targeted approaches in order to minimize disease recurrence.

In ccRCC, de novo lipogenesis is highly activated compared to normal cells $(14,32)$. Of the lipid metabolic genes deregulated in ccRCC, we found SCD1 expression to be consistently amplified in nearly all patient samples across all stages of disease. Selective targeting of SCD1 decreased tumor cell proliferative capacity and promoted apoptosis while producing no notable effects in NRE cells, thereby making SCD1 an ideal candidate for therapeutic intervention in patients presenting with early and late stage ccRCC. Additionally, SCD1 itself may serve as a predictive biomarker, where positive SCD1 IHC would identify appropriate candidate patients who would likely yield a response to pharmacological inhibition of SCD1.

SCD1 expression has been shown to be upregulated in numerous neoplastic lesions including lung adenocarcinoma, non-small cell lung, hypopharyngeal, gastric, breast, prostate, ovary, and colon carcinoma (33-36). Previous experimentation has produced promising results in numerous in vitro and in one pre-clinical experiment demonstrating reduction of tumor volume in a gastric carcinoma model in response to small molecule inhibition of SCD1(34). SCD1 has therefore been proposed as a molecular target for multiple tumor types, whose list now includes ccRCC. While SCD1 expression in normal cells appears to be well characterized, how tumor cells override conventional regulations in order to induce its expression still remains to be clarified.

Mechanistically, inhibition of SCD1 activity revealed increased expression of ER stress markers consistent with the activation of the UPR pathway, suggesting that SCD1 activity positively regulates ER homeostasis. These results are consistent with previous findings in an in vitro setting $(34,37)$, and our group has now confirmed this in vivo with our ccRCC tumor xenograft model. Additionally, our work mechanistically links ER stress induction due to SCD1 inhibition as the mediator of apoptosis, and may therefore serve as a practical biomarker for response to anti-SCD1 therapy. The UPR is characterized by signaling through core ER stress sensors ATF6, PERK (PKR-like ER kinase), and IRE1 (Inositolrequiring enzyme-1) (38). These central signal transducers regulate several responses downstream including: transcription of molecular chaperones, ubiquitin ligase activity, 
attenuation of translation, as well as activation of other protective or pro-apoptotic factors. These reactions all result in either the restoration of cellular homeostasis or cell death in prolonged or severe cases of stress $(39,40)$. Previous studies have linked SFA accumulation to induction of ER stress $(41,42)$, and we believe that loss of SCD1 activity plays a role in this process although the mechanism of de novo lipid biosynthesis in conjunction with ER stress needs to be further investigated. Current literature suggests that increased exposure of cells to SFAs corresponds to an accumulation of SFA content in membrane structures, altering the morphology and decreasing membrane fluidity (43). This may compromise the integrity as well as the functionality of the membranes, including those of the ER, leading to a stress response (43-45). Desaturation of fatty acids is thought to counter these effects, and is protective against SFA mediated stress $(43,46,47)$. This proposed mechanism is summarized in Figure 6D.

In order to address the notion of utilizing multi-targeted therapy as a more effective strategy for treatment of ccRCC, combinatorial options were examined pairing A939572 with current standard-of-care regimens. Previous experimentation targeting SCD1 pharmacologically has demonstrated minimal adverse side effects, and this suggests a reduced risk of compounding drug toxicity when used in combinatorial therapy. Those reported using animal models include dry eye, squinting, and alopecia; which proved to be reversible upon discontinuation of treatment $(48,49)$. Observations from our own in vivo study using an SCD1 inhibitor corroborate these findings. Of note, given the role of SCD1 in lipid metabolism, it is currently being investigated as an attractive target for the treatment of diabetes, obesity, and other metabolic diseases; however detailed effects of SCD1 inhibition on metabolism as well as toxic effects in humans remain to be completely defined $(48,50)$. One clinical trial investigating the use of a liver-targeting SCD1 inhibitor MK-8245(48) for treatment of type II diabetes produced no serious adverse events in all patients evaluated (clinical trial identifier NCT00790556). Our investigations identified a robust synergistic combinatorial response in ccRCC cells both in vitro and in vivo to A939572 paired with temsirolimus. The exact mechanism of this synergistic collaboration is not well defined, and further studies must be performed. Also, while we did not observe synergistic inhibition of tumor cell growth utilizing A939572 in combination with approved TKIs, we believe that this course of therapy warrants deeper investigation due to the limitations of examining drug effects in vitro versus that of animal models where angiogenesis plays key role in tumor growth.

In conclusion our findings support that increased SCD1 expression is tumor specific in ccRCC, and is maintained throughout the course of disease progression. Genetic and molecular targeting of SCD1 activity results in tumor specific inhibition of cell growth and induction of apoptosis both in vitro and in vivo, which is mediated by the ER stress response. Finally, combined treatment of ccRCC with A939572 and an mTOR inhibitor resulted in synergistic inhibition of tumor growth. Taken together, we propose SCD1 as a novel molecular target for the treatment of ccRCC which should be investigated as a therapeutic option alone or in combination with mTOR inhibitors for patients suffering from advanced or metastatic ccRCC.

\section{Supplementary Material}

Refer to Web version on PubMed Central for supplementary material.

\section{Acknowledgments}

Grant and Financial Support:

This work was funded in part from NIH/NCI grants R01CA104505, R01CA136665, R01CA104505-05S1 (JAC); a generous gift from the David \& Lois Stulberg Endowed Fund for Kidney Cancer Research (JAC); Mr. and Mrs. 
Ompal Chauhan Research Fund; Kidney Cancer Research at Mayo Clinic in Florida (JAC); James C. and Sarah K. Kennedy Mayo Clinic Research Career Development Award for Clinicians (HWT); Scheidel Foundation (JAC); Fraternal Order of Eagles Florida State Auxiliary (JAC) and a grant for rare cancers from Dr. Ellis and Dona Brunton (JAC).

We thank Brandy Edenfield for processing and staining immunohistochemistry tissues. We also thank Dr. David Katzman for careful reading of the manuscript, and Hege Ekeberg Larsen and Austin Rohl for their technical support. We are appreciative to the Mayo Clinic Nephrectomy Registry Oversight Group for allowing the use of renal tissues in this study and the Mayo Clinic Biospecimens Accessioning and Processing Laboratory for processing the frozen tissues.

\section{Abbreviations}

$\begin{array}{ll}\text { SCD1 } & \text { Stearoyl CoA desaturase 1 } \\ \text { ccRCC } & \text { clear cell renal cell carcinoma } \\ \text { MUFA } & \text { monounsaturated fatty acid } \\ \text { SFA } & \text { saturated fatty acid } \\ \text { OA } & \text { oleic acid } \\ \text { BSA } & \text { bovine serum albumin } \\ \text { ER } & \text { endoplasmic reticulum } \\ \text { UPR } & \text { unfolded protein response } \\ \text { TKI } & \text { tyrosine kinase inhibitor }\end{array}$

\section{References}

1. Chow WH, Dong LM, Devesa SS. Epidemiology and risk factors for kidney cancer. Nat Rev Urol. 2010; 7:245-57. [PubMed: 20448658]

2. Erdogan F, Demirel A, Polat O. Prognostic significance of morphologic parameters in renal cell carcinoma. Int J Clin Pract. 2004; 58:333-6. [PubMed: 15161115]

3. Coppin C, Kollmannsberger C, Le L, Porzsolt F, Wilt TJ. Targeted therapy for advanced renal cell cancer (RCC): a Cochrane systematic review of published randomised trials. BJU Int. 2011; 108:1556-63. [PubMed: 21952069]

4. Fisher RI, Rosenberg SA, Fyfe G. Long-term survival update for high-dose recombinant interleukin-2 in patients with renal cell carcinoma. Cancer J Sci Am. 2000; 6 (Suppl 1):S55-7. [PubMed: 10685660]

5. Clark PE. The role of VHL in clear-cell renal cell carcinoma and its relation to targeted therapy. Kidney Int. 2009; 76:939-45. [PubMed: 19657325]

6. Haddad H, Rini BI. Current treatment considerations in metastatic renal cell carcinoma. Curr Treat Options Oncol. 2012; 13:212-29. [PubMed: 22410708]

7. Motzer RJ, Hutson TE, Tomczak P, Michaelson MD, Bukowski RM, Rixe O, et al. Sunitinib versus interferon alfa in metastatic renal-cell carcinoma. N Engl J Med. 2007; 356:115-24. [PubMed: 17215529]

8. Sternberg CN, Davis ID, Mardiak J, Szczylik C, Lee E, Wagstaff J, et al. Pazopanib in locally advanced or metastatic renal cell carcinoma: results of a randomized phase III trial. J Clin Oncol. 2010; 28:1061-8. [PubMed: 20100962]

9. Escudier B, Eisen T, Stadler WM, Szczylik C, Oudard S, Siebels M, et al. Sorafenib in advanced clear-cell renal-cell carcinoma. N Engl J Med. 2007; 356:125-34. [PubMed: 17215530]

10. Rini BI, Escudier B, Tomczak P, Kaprin A, Szczylik C, Hutson TE, et al. Comparative effectiveness of axitinib versus sorafenib in advanced renal cell carcinoma (AXIS): a randomised phase 3 trial. Lancet. 2011; 378:1931-9. [PubMed: 22056247] 
11. Patel PH, Senico PL, Curiel RE, Motzer RJ. Phase I study combining treatment with temsirolimus and sunitinib malate in patients with advanced renal cell carcinoma. Clin Genitourin Cancer. 2009; 7:24-7. [PubMed: 19213664]

12. Negrier S, Gravis G, Perol D, Chevreau C, Delva R, Bay JO, et al. Temsirolimus and bevacizumab, or sunitinib, or interferon alfa and bevacizumab for patients with advanced renal cell carcinoma (TORAVA): a randomised phase 2 trial. Lancet Oncol. 2011; 12:673-80. [PubMed: 21664867]

13. Jonasch E, Futreal PA, Davis IJ, Bailey ST, Kim WY, Brugarolas J, et al. State of the science: an update on renal cell carcinoma. Mol Cancer Res. 2012; 10:859-80. [PubMed: 22638109]

14. Tun HW, Marlow LA, von Roemeling CA, Cooper SJ, Kreinest P, Wu K, et al. Pathway signature and cellular differentiation in clear cell renal cell carcinoma. PLoS One. 2010; 5:e10696. [PubMed: 20502531]

15. Igal RA. Stearoyl-CoA desaturase-1: a novel key player in the mechanisms of cell proliferation, programmed cell death and transformation to cancer. Carcinogenesis. 2010; 31:1509-15. [PubMed: 20595235]

16. Aguilar PS, de Mendoza D. Control of fatty acid desaturation: a mechanism conserved from bacteria to humans. Mol Microbiol. 2006; 62:1507-14. [PubMed: 17087771]

17. Ntambi JM. Regulation of stearoyl-CoA desaturase by polyunsaturated fatty acids and cholesterol. J Lipid Res. 1999; 40:1549-58. [PubMed: 10484602]

18. Cooper SJ, Von Roemeling CA, Kang KH, Marlow LA, Grebe SK, Menefee ME, et al. Reexpression of tumor suppressor, sFRP1, leads to antitumor synergy of combined HDAC and methyltransferase inhibitors in chemoresistant cancers. Mol Cancer Ther. 2012

19. Schmittgen TD, Livak KJ. Analyzing real-time PCR data by the comparative C(T) method. Nat Protoc. 2008; 3:1101-8. [PubMed: 18546601]

20. Copland JA, Marlow LA, Kurakata S, Fujiwara K, Wong AK, Kreinest PA, et al. Novel highaffinity PPARgamma agonist alone and in combination with paclitaxel inhibits human anaplastic thyroid carcinoma tumor growth via p21WAF1/CIP1. Oncogene. 2006; 25:2304-17. [PubMed: 16331265]

21. Chou, TC.; Hayball, MP. Biosoft. Cambridge (UK): 1997. CalcuSyn for Windows: multiple-drug dose effect analyzer and manual.

22. Xin Z, Zhao H, Serby MD, Liu B, Liu M, Szczepankiewicz BG, et al. Discovery of piperidine-aryl urea-based stearoyl-CoA desaturase 1 inhibitors. Bioorg Med Chem Lett. 2008; 18:4298-302. [PubMed: 18632269]

23. Dombroski BA, Nayak RR, Ewens KG, Ankener W, Cheung VG, Spielman RS. Gene expression and genetic variation in response to endoplasmic reticulum stress in human cells. Am J Hum Genet. 2010; 86:719-29. [PubMed: 20398888]

24. Wang Y, Shen J, Arenzana N, Tirasophon W, Kaufman RJ, Prywes R. Activation of ATF6 and an ATF6 DNA binding site by the endoplasmic reticulum stress response. J Biol Chem. 2000; 275:27013-20. [PubMed: 10856300]

25. Healy SJ, Gorman AM, Mousavi-Shafaei P, Gupta S, Samali A. Targeting the endoplasmic reticulum-stress response as an anticancer strategy. Eur J Pharmacol. 2009; 625:234-46. [PubMed: 19835867]

26. Schroder M, Kaufman RJ. The mammalian unfolded protein response. Annu Rev Biochem. 2005; 74:739-89. [PubMed: 15952902]

27. Chou TC, Talalay P. Analysis of Combined Drug Effects - a New Look at a Very Old Problem. Trends Pharmacol Sci. 1983; 4:450-4.

28. Luyimbazi D, Akcakanat A, McAuliffe PF, Zhang L, Singh G, Gonzalez-Angulo AM, et al. Rapamycin regulates stearoyl CoA desaturase 1 expression in breast cancer. Mol Cancer Ther. 2010; 9:2770-84. [PubMed: 20876744]

29. Hanahan D, Weinberg RA. Hallmarks of cancer: the next generation. Cell. 2011; 144:646-74. [PubMed: 21376230]

30. Tan DS, Gerlinger M, Teh BT, Swanton C. Anti-cancer drug resistance: understanding the mechanisms through the use of integrative genomics and functional RNA interference. Eur J Cancer. 2010; 46:2166-77. [PubMed: 20413300] 
31. Ebos JM, Lee CR, Kerbel RS. Tumor and host-mediated pathways of resistance and disease progression in response to antiangiogenic therapy. Clin Cancer Res. 2009; 15:5020-5. [PubMed: 19671869]

32. Pinthus JH, Whelan KF, Gallino D, Lu JP, Rothschild N. Metabolic features of clear-cell renal cell carcinoma: mechanisms and clinical implications. Can Urol Assoc J. 2011; 5:274-82. [PubMed: 21801687]

33. Scaglia N, Chisholm JW, Igal RA. Inhibition of stearoylCoA desaturase-1 inactivates acetyl-CoA carboxylase and impairs proliferation in cancer cells: role of AMPK. PLoS One. 2009; 4:e6812. [PubMed: 19710915]

34. Roongta UV, Pabalan JG, Wang X, Ryseck RP, Fargnoli J, Henley BJ, et al. Cancer cell dependence on unsaturated fatty acids implicates stearoyl-CoA desaturase as a target for cancer therapy. Mol Cancer Res. 2011; 9:1551-61. [PubMed: 21954435]

35. Mason P, Liang B, Li L, Fremgen T, Murphy E, Quinn A, et al. SCD1 inhibition causes cancer cell death by depleting mono-unsaturated fatty acids. PLoS One. 2012; 7:e33823. [PubMed: 22457791]

36. Hess D, Chisholm JW, Igal RA. Inhibition of stearoylCoA desaturase activity blocks cell cycle progression and induces programmed cell death in lung cancer cells. PLoS One. 2010; 5:e11394. [PubMed: 20613975]

37. Minville-Walz M, Pierre AS, Pichon L, Bellenger S, Fevre C, Bellenger J, et al. Inhibition of stearoyl-CoA desaturase 1 expression induces CHOP-dependent cell death in human cancer cells. PLoS One. 2010; 5:e14363. [PubMed: 21179554]

38. Samali A, Fitzgerald U, Deegan S, Gupta S. Methods for monitoring endoplasmic reticulum stress and the unfolded protein response. Int J Cell Biol. 2010; 2010:830307. [PubMed: 20169136]

39. Xu C, Bailly-Maitre B, Reed JC. Endoplasmic reticulum stress: cell life and death decisions. J Clin Invest. 2005; 115:2656-64. [PubMed: 16200199]

40. Walter P, Ron D. The unfolded protein response: from stress pathway to homeostatic regulation. Science. 2011; 334:1081-6. [PubMed: 22116877]

41. Wei Y, Wang D, Topczewski F, Pagliassotti MJ. Saturated fatty acids induce endoplasmic reticulum stress and apoptosis independently of ceramide in liver cells. Am J Physiol Endocrinol Metab. 2006; 291:E275-81. [PubMed: 16492686]

42. Wang D, Wei Y, Pagliassotti MJ. Saturated fatty acids promote endoplasmic reticulum stress and liver injury in rats with hepatic steatosis. Endocrinology. 2006; 147:943-51. [PubMed: 16269465]

43. Deguil J, Pineau L, Rowland Snyder EC, Dupont S, Beney L, Gil A, et al. Modulation of lipidinduced ER stress by fatty acid shape. Traffic. 2011; 12:349-62. [PubMed: 21143717]

44. Borradaile NM, Han X, Harp JD, Gale SE, Ory DS, Schaffer JE. Disruption of endoplasmic reticulum structure and integrity in lipotoxic cell death. J Lipid Res. 2006; 47:2726-37. [PubMed: 16960261]

45. Basseri S, Austin RC. Endoplasmic reticulum stress and lipid metabolism: mechanisms and therapeutic potential. Biochem Res Int. 2012; 2012:841362. [PubMed: 22195283]

46. Miller TA, LeBrasseur NK, Cote GM, Trucillo MP, Pimentel DR, Ido Y, et al. Oleate prevents palmitate-induced cytotoxic stress in cardiac myocytes. Biochem Biophys Res Commun. 2005; 336:309-15. [PubMed: 16126172]

47. Welters HJ, Tadayyon M, Scarpello JH, Smith SA, Morgan NG. Mono-unsaturated fatty acids protect against beta-cell apoptosis induced by saturated fatty acids, serum withdrawal or cytokine exposure. FEBS Lett. 2004; 560:103-8. [PubMed: 14988006]

48. Oballa RM, Belair L, Black WC, Bleasby K, Chan CC, Desroches C, et al. Development of a livertargeted stearoyl-CoA desaturase (SCD) inhibitor (MK-8245) to establish a therapeutic window for the treatment of diabetes and dyslipidemia. J Med Chem. 2011; 54:5082-96. [PubMed: 21661758]

49. Li CS, Belair L, Guay J, Murgasva R, Sturkenboom W, Ramtohul YK, et al. Thiazole analog as stearoyl-CoA desaturase 1 inhibitor. Bioorg Med Chem Lett. 2009; 19:5214-7. [PubMed: 19632834]

50. Uto Y, Ogata T, Kiyotsuka Y, Miyazawa Y, Ueno Y, Kurata H, et al. Novel and potent inhibitors of stearoyl-CoA desaturase-1. Part II: Identification of 4-ethylamino-3-(2-hydroxyethoxy)-N-[5- 
(3-trifluoromethylbenzyl)thiazol-2-yl]benzamide and its biological evaluation. Bioorg Med Chem Lett. 2009; 19:4159-66. [PubMed: 19541482] 


\section{Translational Relevance}

Presently, there is a paucity of effective therapies designed to target clear cell renal cell carcinoma (ccRCC) effectuating long term durable response in patients with advanced disease. Additionally, there is a lack of molecular markers that can be remedially targeted, demonstrating tumor specific inhibition. Therefore, current therapeutic approaches often produce adverse side effects. We examined matched diseased and normal gene expression signatures, and identified that Stearoyl-CoA desaturase 1 (SCDI) was consistently overexpressed in ccRCC. Investigation of SCD1 as a molecular target for ccRCC intervention yielded antitumor activity in vitro and in vivo via activation of endoplasmic reticulum (ER) stress. Accordingly, induced ER stress proteins may be reliable biomarkers for response to therapy of an SCD1 inhibitor. Moreover, we discovered antitumor synergy with the FDA approved mTOR inhibitor, temsirolimus. This regimen may be a reasonable combinatorial therapy leading to enhanced efficacy and survival over that of current therapies for metastatic ccRCC. 
A.

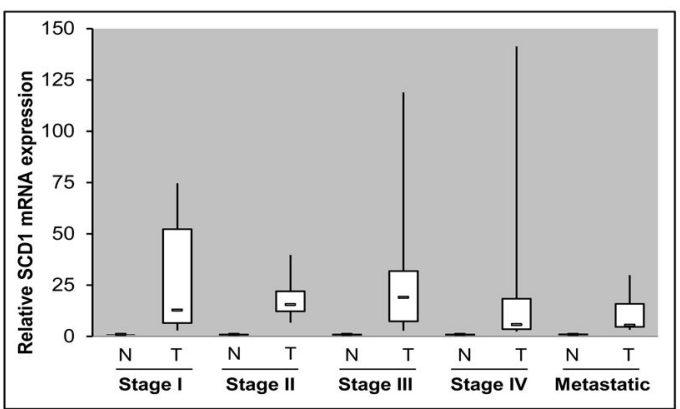

B.
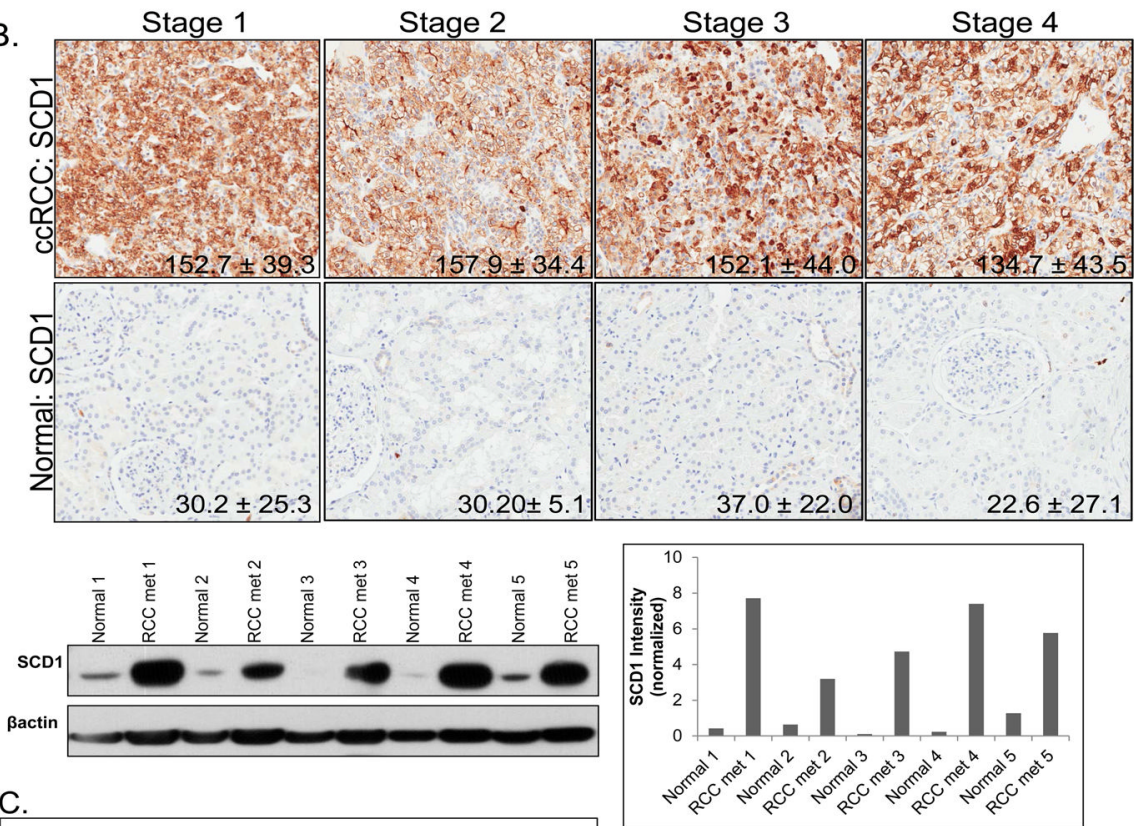

C.

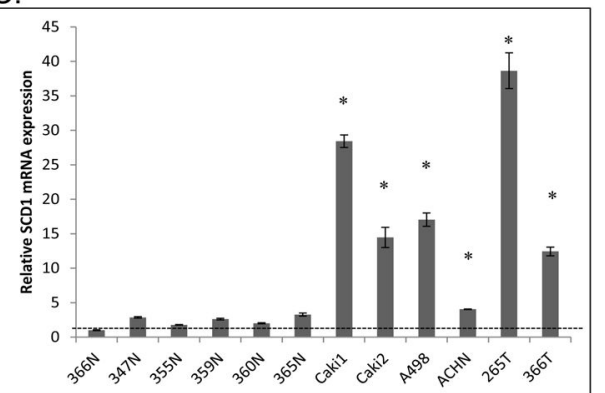

D.

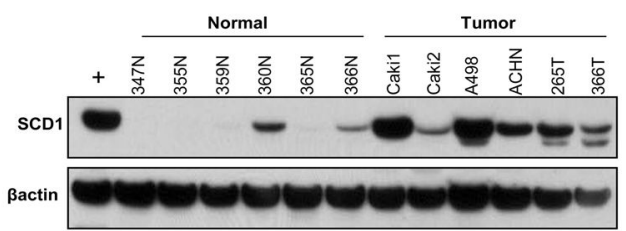

Figure 1.

SCD1 expression is upregulated in ccRCC. (A) SCD1 mRNA expression shown for ccRCC tissue samples relative to matched normal samples across stages I-IV with average fold change increases of $29.7(n=7), 18.0(n=8), 30.0(n=8), 23.9(n=12)$, and $11.5(n=8)$ in stage $1,2,3,4$ and metastatic groups respectively, graphed as a box whisker plot. (B) IHC for SCD1 expression in patient normal and matched ccRCC tissue stage I-IV with average Hscore expression ranging from 134.7 to 157.9 in tumor vs. 22.6 to 37.0 in normal $(n=42,24$, 35,11 in tumor stage $1-4$ and 40,30,37, 5 for normal matches respectively. Western blot analysis and quantitation of SCD1 expression in protein lysates prepared from patient metastatic tissue matched to distant site normal samples. (C) mRNA expression analysis of normal renal epithelial (NRE) versus ccRCC cell lines with fold change inductions of 28.4, 14.4, 17.0, 4.0, 38.6, and 12.4 seen in Caki1, Caki2, A498, ACHN, KIJ265T, and 
RWV366T when normalized to $366 \mathrm{~N}$, which possessed the lowest SCD1 expression and is set at 1. SCD1 expression in NRE's ranged from 1 (in 366N) to 3.2 (in 365N). (D) Western blot for SCD1 protein expression in NRE and ccRCC cell lines. 
A.

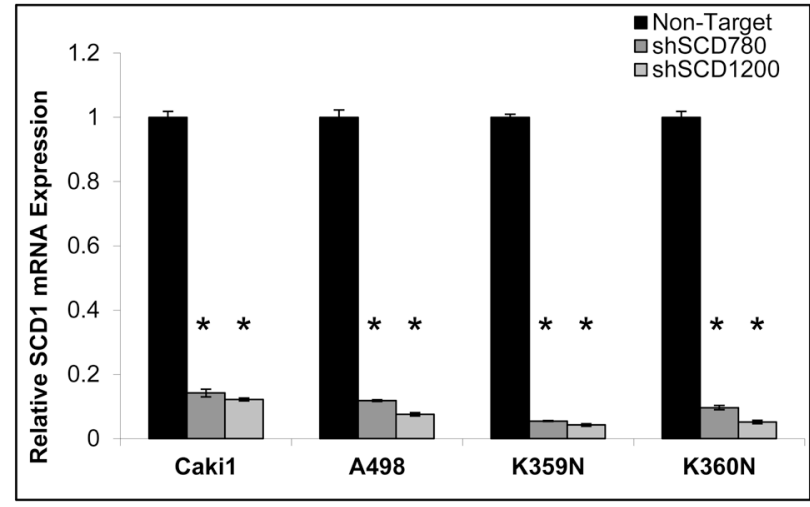

B.

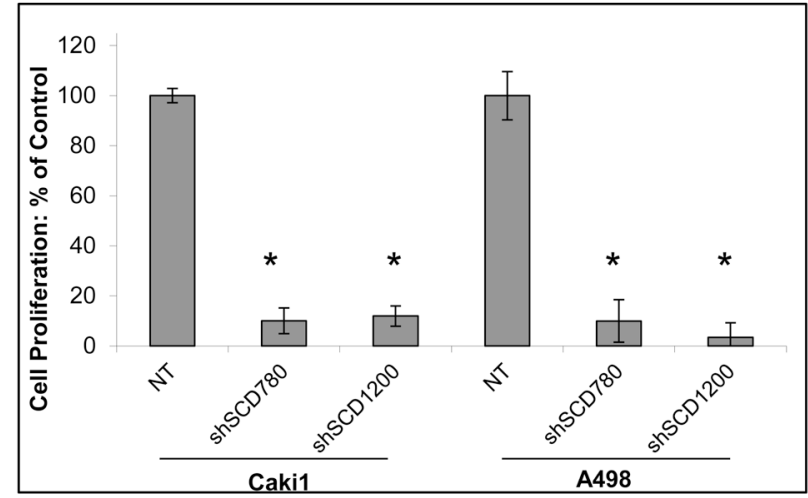

C.

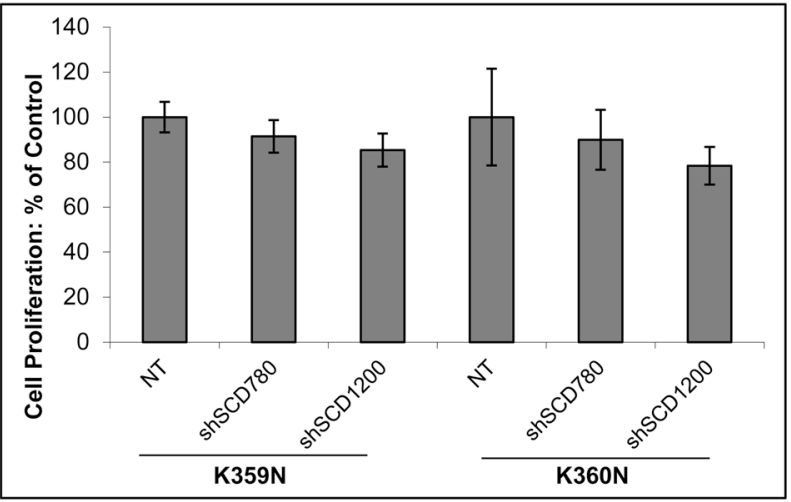

D.

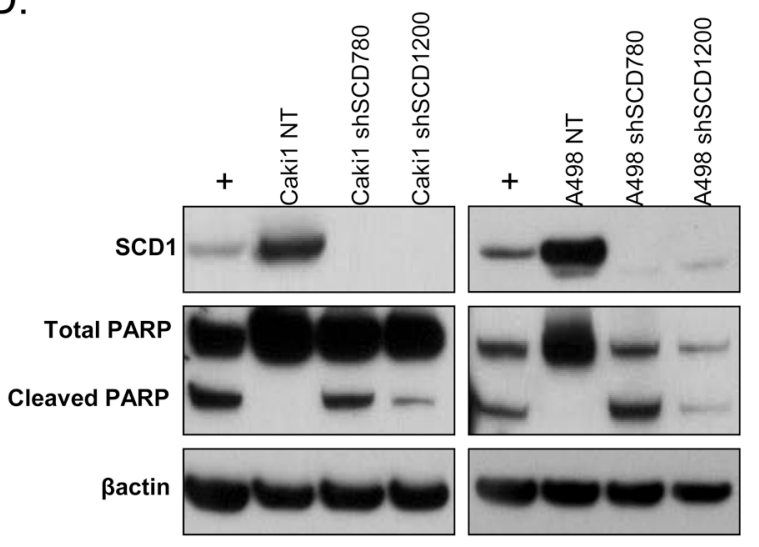

Figure 2.

SCD1 expression in ccRCC is tumor specific and is required for tumor growth and survival. Knockdown of SCD1 in ccRCC as shown by decrease in both (A) mRNA and (D) protein expression using two separate lentiviral constructs shSCD780 and shSCD1200. Proliferation in (B) A498 and Cakil ccRCC cell lines and (C) NRE samples of NT versus shSCD lentiviral infected cells. (D) Immunoblot for Poly-ADP ribose polymerase (PARP) cleavage and SCD1 expression in A498 and Caki1 cell lines. 
A.

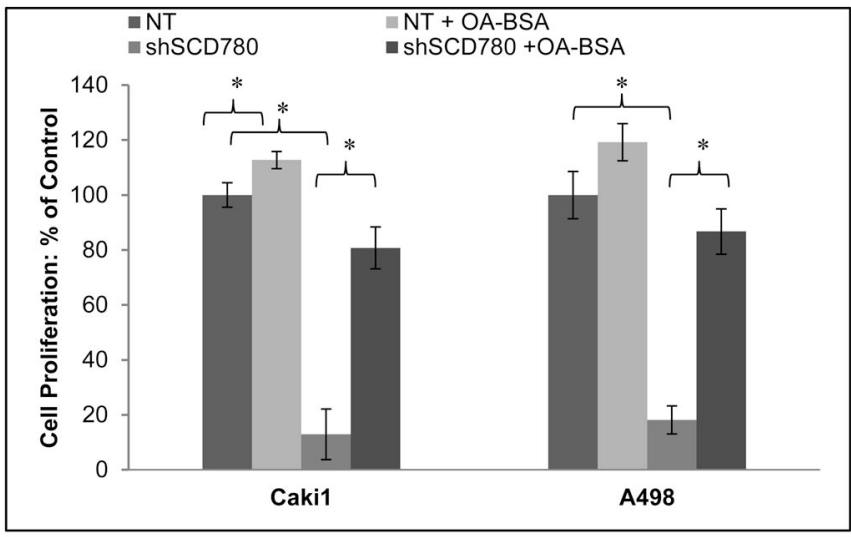

B.

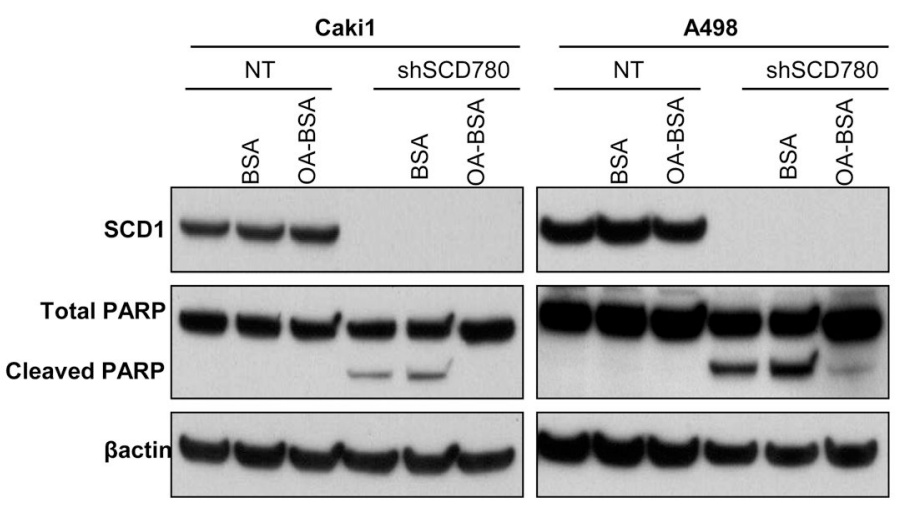

C.

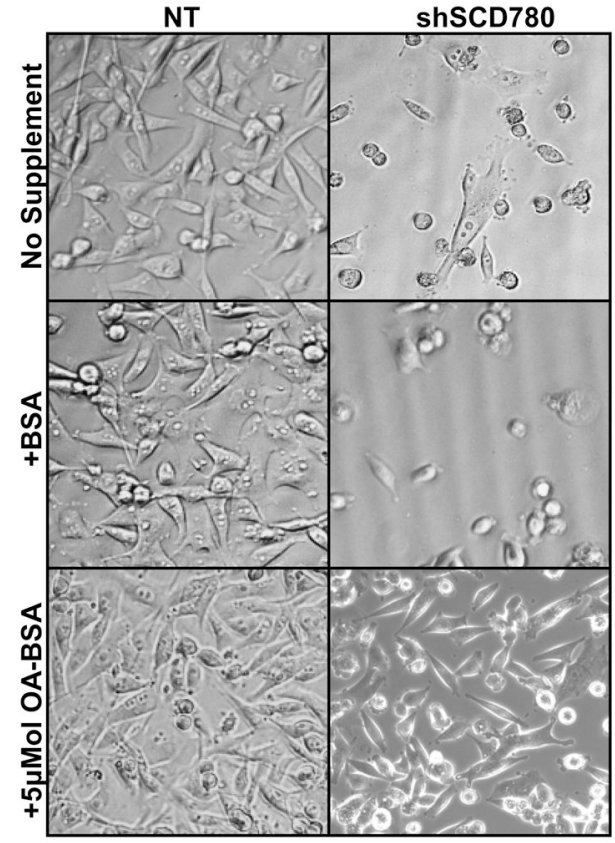

Figure 3.

Anti-proliferative and apoptotic induction via loss of SCD1 expression can be rescued with addition of oleic acid (OA-BSA). (A) Proliferation and (B) western blot analysis for SCD1 and PARP cleavage in Caki1 and A498 NT versus shSCD with or without OA-BSA supplementation. (C) Phase-contrast microscopy displays representative ccRCC cell (Caki1) confluence at day 5 of proliferation assay with different treatment conditions. 
A.

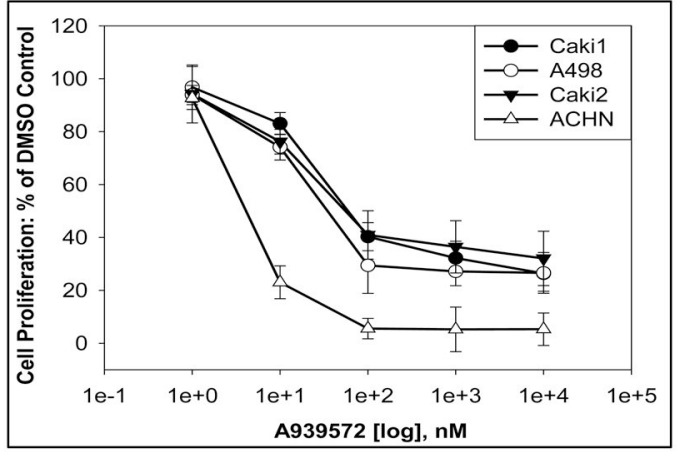

B.

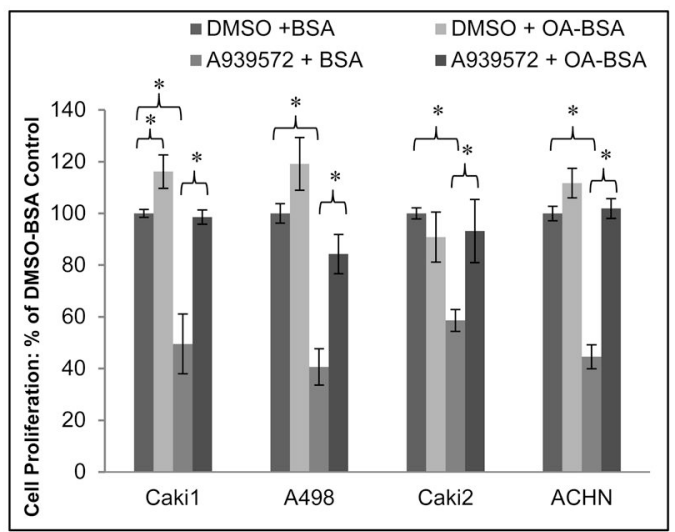

C.

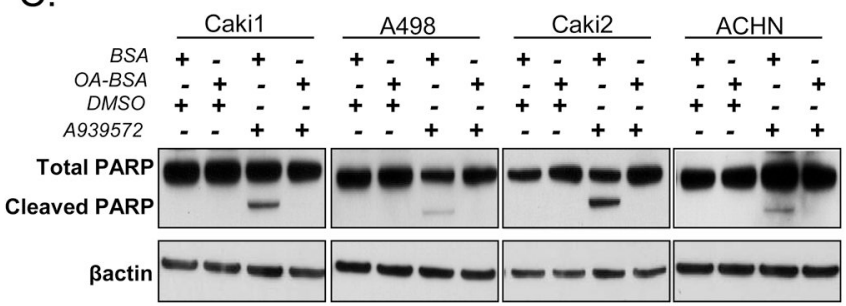

D.

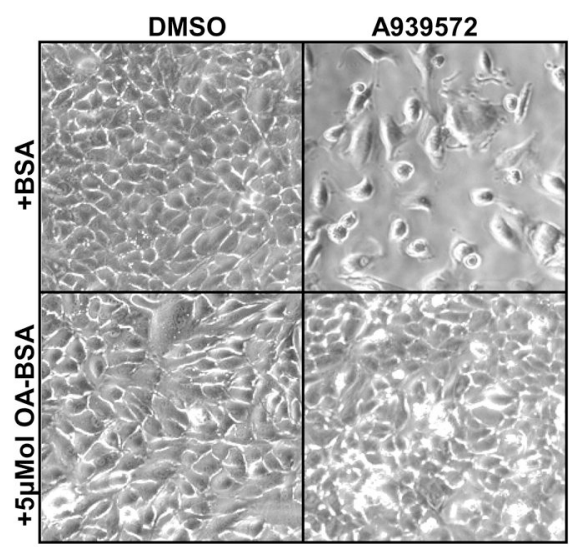

Figure 4.

Treatment of ccRCC cells with a small molecule SCD1 inhibitor, A939572, inhibits cell growth and induces apoptosis. (A) Cell proliferative response to dose out of A939572 in Caki1, A498, Caki2, and ACHN ccRCC cell lines. (B) ccRCC proliferation rescue with OABSA in A939572 treated ccRCC cell lines. (C) Western blot analysis for PARP cleavage in A939572 treated vs. control, as well as OA-BSA rescue in ccRCC cell lines. (D) Representative phase contrast images of A939572 treated ccRCC cells (A498) +/-OA-BSA rescue at day 5 . 
A.
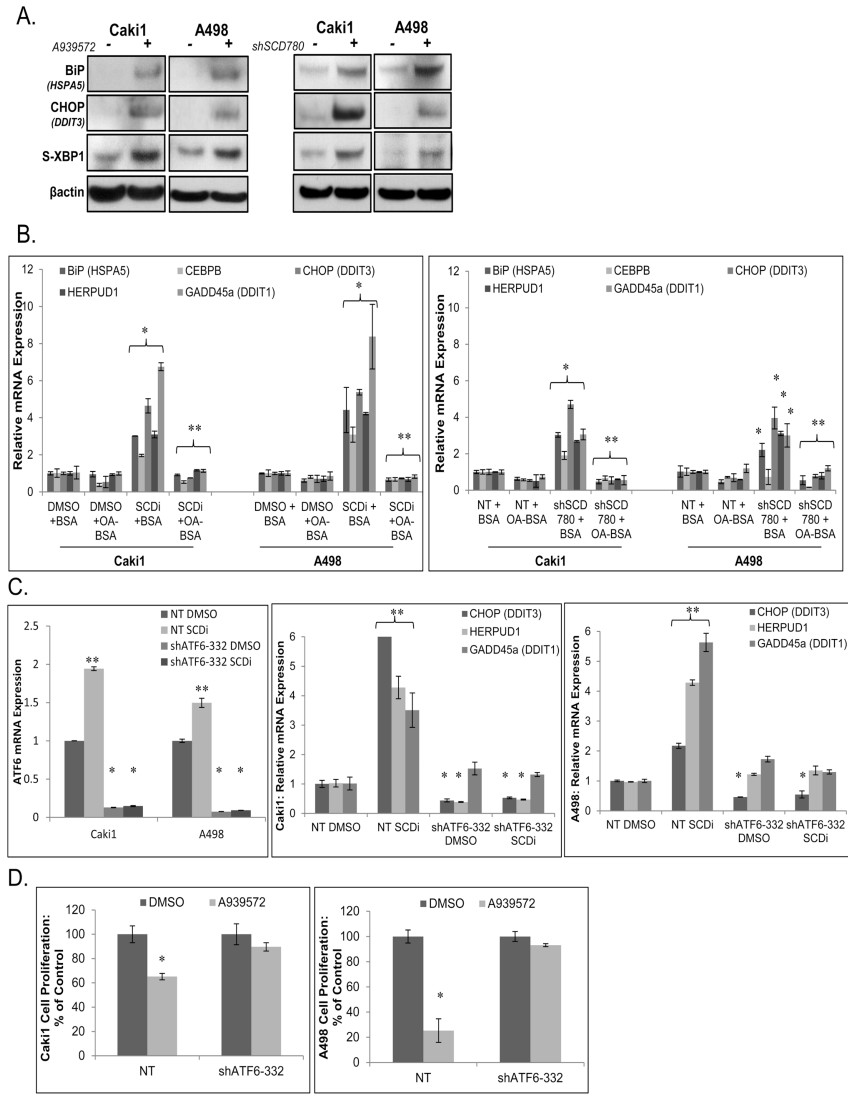

Figure 5.

Inhibition of SCD1 activity in ccRCC induces cell death mediated by endoplasmic reticulum stress response. (A) Western blot analysis for expression of ER stress markers: BiP, CHOP, and spliced XBP1 in response to A939572 treatment or lentiviral silencing of SCD1 in Caki1 and A498. (B) QPCR analysis of ER stress gene expression in Caki1 and A498 cells treated with A939572 (75nM) or shSCD lentivirus +/-OA-BSA rescue after $48 \mathrm{Hr}$. (C) QPCR for ATF6 expression in NT and ATF6 knockdown (shATF6-332) Caki1 and A498 cells treated with DMSO or IC50 dose of A939572 (SCDi) for 48 hours (far left panel).

QPCR for ER stress markers CHOP, HERPUD1, and GADD45a in Caki1 (center panel) and A498 (far right panel) ccRCC cells treated with A939572 (IC50 dose for 48 hours); expression is normalized to NT DMSO sample for all groups. Statistically significant correlations between DMSO and SCDi treated NT or shATF6-332 cells are denoted by double asterisk (**). Statistically significant correlations between NT DMSO and each shATF6-332 DMSO and shATF6-332 SCDi are denoted by single asterisk (*). (D) Proliferation of NT and shATF6-332 Caki1 and A498 RCC cells treated with DMSO control or A939572. Doses utilized are as described in panel (C), and cells were counted $72 \mathrm{H}$ posttreatment. 
A.
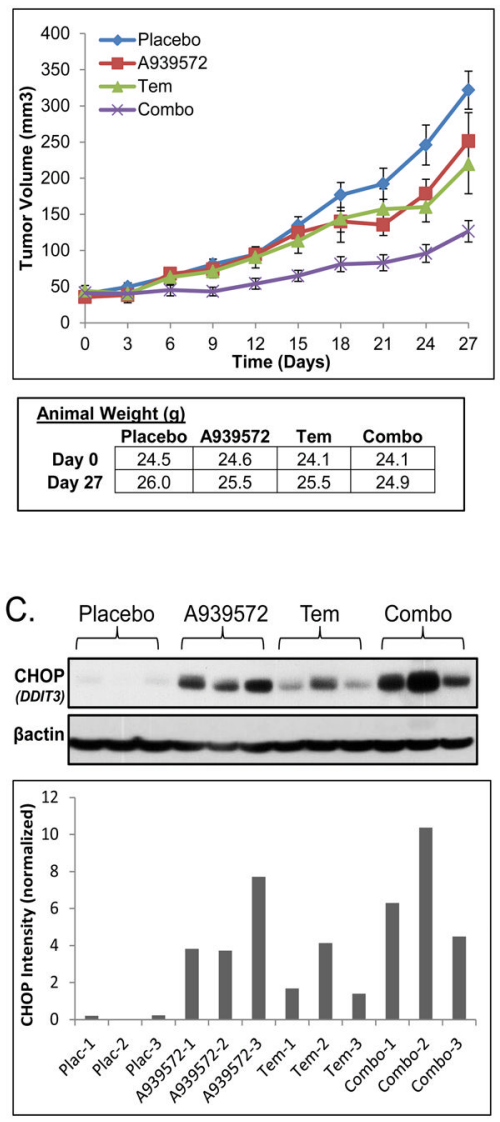

B.

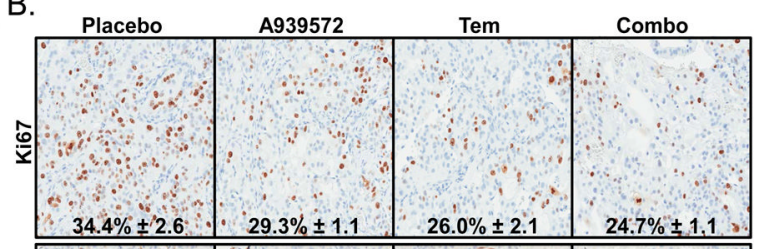

ồ
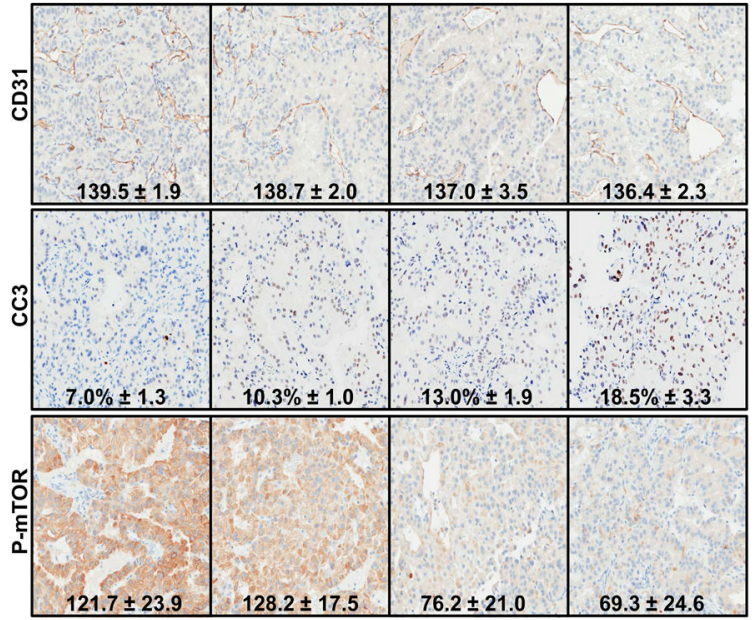

D.

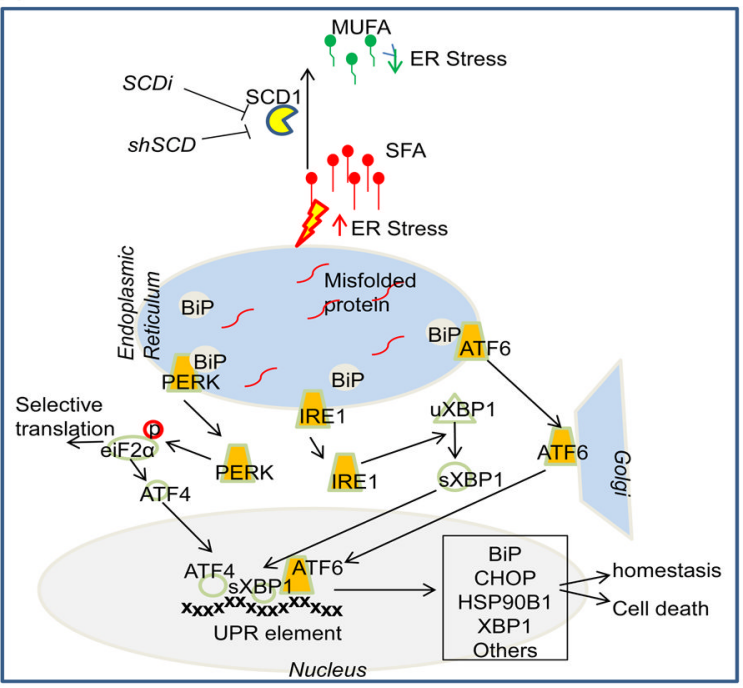

Figure 6.

Treatment of ccRCC cells with SCD1 inhibitor in combination with the mTOR inhibitor Temsirolimus synergistically inhibits tumor cell growth in vivo. (A) In vivo tumor growth analysis and animal weight of A498 ccRCC subcutaneous xenografts in female athymic nude mice treated with A939572 and Temsirolimus alone or in combination versus placebo control ( $\mathrm{n}=10$ per group). (B) IHC of tissue harvested from treatment groups stained for Ki67 and CC3 (quantitated by N-score), CD31 (quantitated by I-score), and phospho-mTOR (quantitated by $\mathrm{H}$-score). Average group scores $+/-$ the standard error are reported for each stain. (C) Western blot and quantitation of CHOP expression in all four treatment groups. (D) Model of proposed SCD1 activity in ccRCC model: inhibition of SCD1 blocks desaturation of SFA resulting in an accumulation of SFA species which trigger the ER stress response. 